\title{
Study of EBSD Experiment Parameters Influence on Computation of Polycrystalline Pole Figures and Orientation Distribution Function
}

\author{
Anastasia O. Antonova ${ }^{1}$, Pavel S. Dzhumaev ${ }^{2}$ and Tatyana I. Savyolova ${ }^{1}$ \\ ${ }^{1}$ Moscow Engineering Physics Institute (State University), Department of Applied Mathematics, Kashirskoe sh. 31, Moscow, 115409 \\ Russia \\ ${ }_{2}^{2}$ Moscow Engineering Physics Institute (State University), Department of Material Science, Kashirskoe sh. 31, Moscow, 115409 Russia
}

\begin{abstract}
Mathematical model for a polycrystalline specimen and EBSD experiment is proposed. As the measurement parameters, the scanning step and the threshold disorientation angle are considered. To study the impact of the measurement parameters Pole Figures and Orientation Distribution Function of model specimen and corresponding ones, calculated from model EBSD measurements, are compared. The real EBSD experiment was also performed. The results of the model experiment are correlated with such detected in the real EBSD data. The most significant results are formulated in the given work.
\end{abstract}

\section{Introduction}

Recent advances in electron back scattering diffraction (EBSD) [1] have opened up new opportunities in the study of material structure and texture. The problem of choosing measurement parameters remains insufficiently investigated, while these parameters have a large effect on measurement results. It was indicated than an unsuitable choice of measurement parameters leads to incorrect results. For example, an insufficient number of orientation measurements results in low accuracy of computed Pole Figures (PFs) [2]; disorientation angle measurements may lead to inconsistent average grain size values [3]; a small step of measurements leads to a considerable increase in time required for conducting an experiment (see [4]). For solving the problem of EBSD parameters choosing we need the opportunity to compare the specimen data and obtained EBSD data. Accordingly, it is of interest to study this issue with the help of the mathematical simulation of an experiment. If the real specimen characteristics are close to ones of the model specimen the EBSD experiment model helps to make a decision about experiment parameters.

Below, we develop a two-dimensional mathematical model of a specimen and a subsequent EBSD experiment. Specimen measurements are modeled for various experiment parameters. PF and orientation distribution functions (ODF) for model specimen and corresponding ones, calculated from model EBSD data, are compared for each set of experiment parameters. The substantial result of analysis are presented. The real EBSD experiment was also performed. The results of the model experiment (see $[5,6])$ are correlated with such detected in the real EBSD data.

\section{Carrying out a model experiment}

A specimen is a set of grains

$$
\left\{\left(x_{i}, g_{i}, \omega_{i}\right) i=1,2, \ldots N-1,\left(x_{N}, g_{N}\right)\right\},
$$

where $x_{i}$ is the size of the $i$ th grain, which is equal to the diameter of a circle whose area is equal to that of the grain's cross section; $g_{i}=\left(\varphi_{i}, \theta_{i}, \psi_{i}\right)$ is the orientation of the $i$ th grain corresponding to the Euler angles $-\pi \leq$ $\varphi_{i}, \psi_{i}<\pi, 0 \leq \theta_{i} \leq \pi \quad ; \quad \omega_{i}=\left(g_{i}, g_{i+1}\right) \quad$ is the disorientation angle between neighboring grains; and $N$ is the number of grains in the specimen. Grain sizes are chosen as random variables distributed satisfying a gamma distribution [5].

A set of grain orientations with a distribution function in the form of central distribution function (CND) [7] with a given parameter $\varepsilon>0$ is generated by the special Monte-Carlo method with the number of convolutions equal to $n=6$. Grains (Eq. 1) are separated by boundaries, which are variables uniformly distributed in $[0,2 \gamma / 10)$, where $\gamma>0$ is such that the area of the boundaries is $10 \%$ of the grains' area.

To simulate the EBSD experiment the following measurement parameters are specified: $h$ is the scanning step, and $\omega_{0}$ is a threshold disorientation angle for determining two different neighboring grains $g_{i}$ and $g_{i+1}$ from the disorientation angle between them.

Measurements are made at the lattice points $\Delta_{k}=$ $k h, k=1,2, \ldots, N_{1}$ on the surface of the simulated specimen. If the point hits the boundary, it is assigned an orientation corresponding to the Euler angles equal to the 
arithmetic mean of the angles of two nearest determined orientations [7].

The resulting orientations are used to calculate the disorientation angles $\widehat{\omega}_{k}=\left(\hat{g}_{k}, \hat{g}_{k+1}\right)$, $k=1, \ldots, N_{1}-1$. If $\widehat{\omega}_{k} \leq \omega_{0}$, then the $k$ th and $(k+1)$ th steps belong to a single grain. If $n$ measurements are inside the $j$ th grain, then the grain size is set equal to $\tilde{x}_{j}=n h$ and the Euler angles are calculated as the arithmetic mean of the corresponding $n$ measurements. If $\widehat{\omega}_{k}>\omega_{0}$, then a new grain begins at the point $(k+1) h$. As a result of the measurements, we obtain a set of grains

$$
\left\{\left(\tilde{x}_{i}, \tilde{g}_{i}, \widetilde{\omega}_{i}\right), i=1,2, \ldots \widetilde{N}-1,\left(\tilde{x}_{\widetilde{N}}, \tilde{g}_{\widetilde{N}}\right)\right\}
$$

\section{Results of the model experiment}

Following the scheme described in Section 2, we modeled specimen, in which the boundaries amount to about $10 \%$ of the total grain area, with the average grain size equal to $2.0 \mu \mathrm{m}$. The number $N$ of grains in a specimen was set equal to 1000. The grain orientations in a specimen were modeled by the special Monte-Carlo method for CND on $S O(3)$ with texture sharpness $\varepsilon=1 / 8$ (see [7]). The following EBSD measurement parameters were chosen for a given specimen: $h=0.5 \mu \mathrm{m}, 1.0 \mu \mathrm{m}, 2.0 \mu \mathrm{m} ; \omega_{0}=5^{\circ}, 10^{\circ}$ and $20^{\circ}$. Below, the results of observations concerning the ODF and PF changing according to EBSD measurement parameters are given.

Firstly, let us emphasize that for ODFs at $\omega_{0}=20^{\circ}$ and, in some cases, for $\omega_{0}=10^{\circ}$, the maximum is observed to increase [5]. This behavior of the ODF is similar to that in the presence of dependent orientations [7], which arise at $\omega_{0}=20^{\circ}$ because of merging a large number of grains. What about the coordinates of the maximum we can not reasonably confirm whether they remain unchanged or not while the variable $\psi$ is fixed. In the cased of strongly textured specimens, the ODF should be recovered with a small threshold disorientation angle. For the specimens under consideration, as such an angle, we can use $\omega_{0}=5^{\circ}$.

A decrease in the scanning step insignificantly improves the ODF recovered from individual orientations. At the same time, the statistical sample is reduced substantially. For more detail on the effect of a sample on ODFs recovered from individual orientations, see [8].

Some more remarks have to be made about the scanning step. To obtain more accurate shapes and sizes
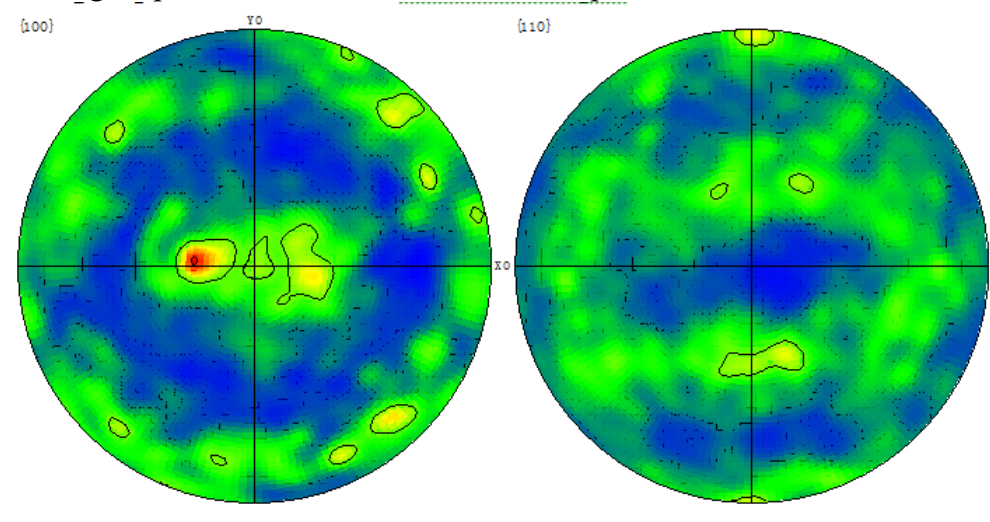
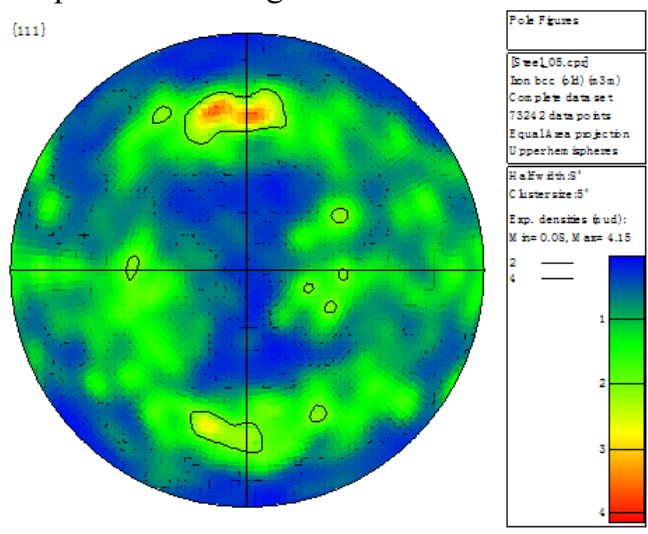

of grains, the step is usually chosen to be $1 / 10-1 / 5$ times as large as the average grain size in the specimen (see [8]). This step corresponds to $\delta_{\text {less_h }}<5 \%$, where $\delta_{\text {less_h }}$ is the fraction of grains whose sizes are less than scanning step. acceptable results are obtained even with a step corresponding to $\delta_{\text {less } \_} \approx 25 \%$ [5]. In this case, the sample turns out to be several times as large.

According to results of specimen PF and PF of modeling measurements comparing it was obtained that for strongly textured specimens threshold disorientation angle is appeared to be more significant than scanning step; for weakly textured specimens, conversely, measurements are strongly affected by the scanning step [6]. The calculated deviation of measured PF from model specimen $\mathrm{Pf}$ in $L_{1}$ metrics and RP-factor for measured PF verify statement mentioned. We should also notice that the PF maximum and its position are unstable to EBSD measurements parameters. Instability grows with weakening of specimen texture sharpness. it shows up in the shift of PF maximum position or second maximum appearing.

The more grain size distribution approaches the uniform one, the stronger instability and the less accurate PF calculation [6].

\section{Correlation of the model experiment results with real EBSD data}

Material for the investigation was a ferritic-martensitic steel EP823 (12Cr-Mo-W-Si-V-Nb-B) treated by the nitrogen gas high-temperature pulsed plasma flows (HTPPF). HTPPF surface modification were carried out by means of the Z-pinch type experimental plasma installation "Desna-M" [9]. This treatment results in the formation of the microcrystalline surface layer with a mean grain size of $2 \mathrm{~mm}$. This layer has a single phase bcc type (a-Fe) equiaxed grain structure with a homogeneous distribution of alloying elements. An EBSD data were acquired using the HKL CHANNEL5 software (Oxford Instruments, UK) coupled to an EVO 50 XVP scanning electron microscope (ZEISS, Germany)..

EBSD measurement were performed with scanning step sizes $h=0.5 \mu \mathrm{m}, 1.0 \mu \mathrm{m}, 2.0 \mu \mathrm{m}$ and then for each step size meaning the different threshold disorientation angle meanings were considered: $\omega_{0}=5^{\circ}, 10^{\circ}$ and $20^{\circ}$.

Below, the results of analysis of the obtained ODF and PF from EBSD orientation data according to EBSD measurement parameters are given. 

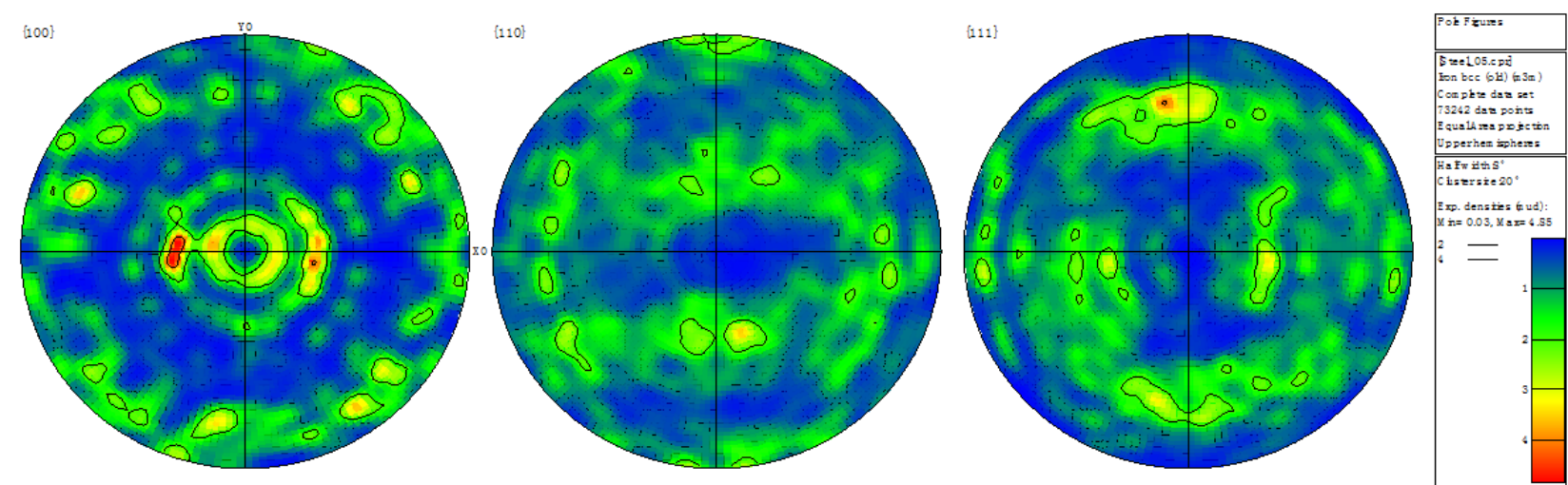

Figure 1. PF for fixed $h=0.5 \mu \mathrm{m}$, (a) for $\omega_{0}=5^{\circ}$ and (b) for $\omega_{0}=20^{\circ}$

First of all, it is worth noting that according to ODF and $\mathrm{PF}$ images measurements are strongly affected by the threshold disorientation angle (Fig. 1), the influence of step size on ODF and PF evaluation is not so significant (Fig. 2). The given statement is in good agreement with model experiment results.

Fig. 1 shows that $\omega_{0}$ can change PF plot essentially. It should be noted that considerably different scanning steps $h=2.0 \mu \mathrm{m}$ and $0.5 \mu \mathrm{m}$ give close PF plots (Fig.2). Herewith the time expended in EBSD measurement increases by a factor of several tens under step size growth (Table 1).

Table. 1. Measurement time

\begin{tabular}{|c|c|c|}
\hline$h[\mu \mathrm{m}]$ & $\begin{array}{c}\text { Number of } \\
\text { points measured }\end{array}$ & $t_{\text {meas }}[\mathrm{h}]$ \\
\hline 2.0 & 4524 & 0.16 \\
\hline 1.0 & 18251 & 0.63 \\
\hline 0.5 & 73242 & 2.56 \\
\hline
\end{tabular}
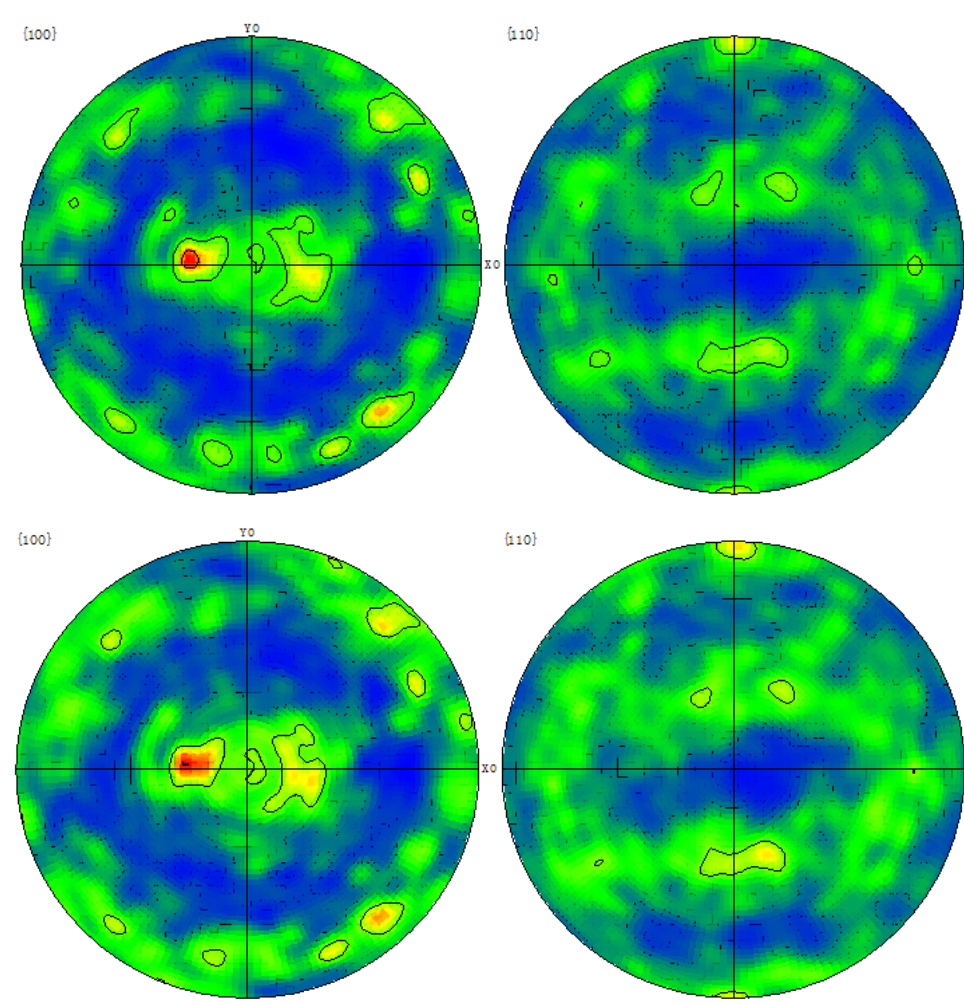

Figure 2. PF for fixed $\omega_{0}=10^{\circ}$, (a) for $h=2.0 \mu \mathrm{m}$ and (b) for $h=0.5 \mu \mathrm{m}$
The behavior of PF and ODF maximums was also considered. Table 2 presents PF maximums according to different EBSD parameters.

Table 2. Meanings of PF maximums

\begin{tabular}{|c|c|c|c|}
\hline \multirow{2}{*}{$h[\mu \mathrm{m}]$} & \multicolumn{3}{|c|}{$\omega_{0}\left[{ }^{\circ}\right]$} \\
\cline { 2 - 4 } & $20^{\circ}$ & $10^{\circ}$ & $5^{\circ}$ \\
\hline 2.0 & 5.56 & 4.85 & 4.82 \\
\hline 1.0 & 4.93 & 4.11 & 4.23 \\
\hline 0.5 & 4.85 & 4.02 & 4.15 \\
\hline
\end{tabular}

Let us notice that PF maximum increases under step size growth for each fixed $\omega_{0}$, but dependence PF maximum on threshold disorientation angle is appeared not so clear: maximum can increase or decrease with $\omega_{0}$ growth. Such behavior points out instability of $\mathrm{PF}$ calculation to EBSD parameters that is obtained in model experiment (Section 1).

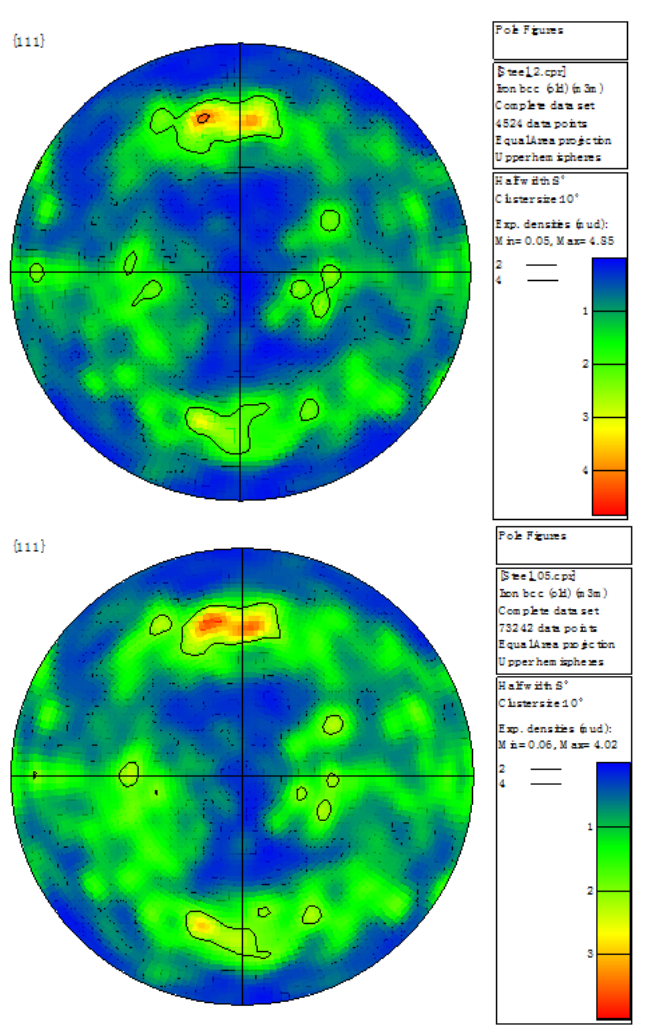




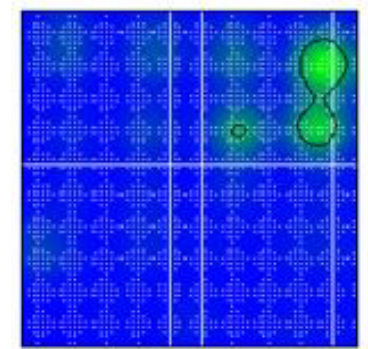

$2-45^{\circ}$

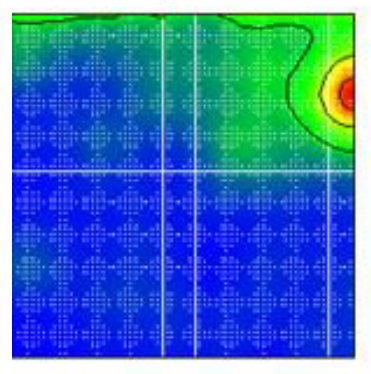

$2-45^{\circ}$

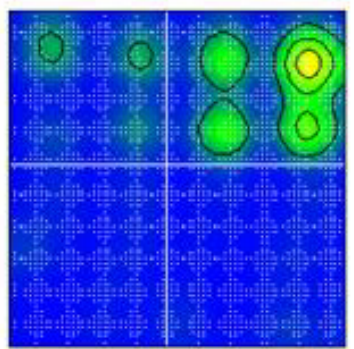

$2-50^{\circ}$

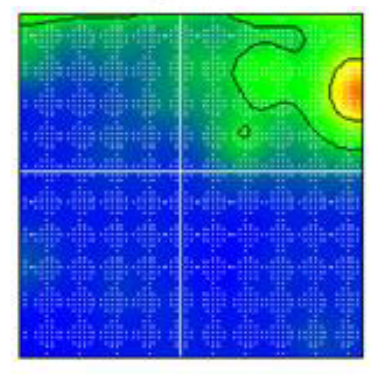

$2-50^{\circ}$
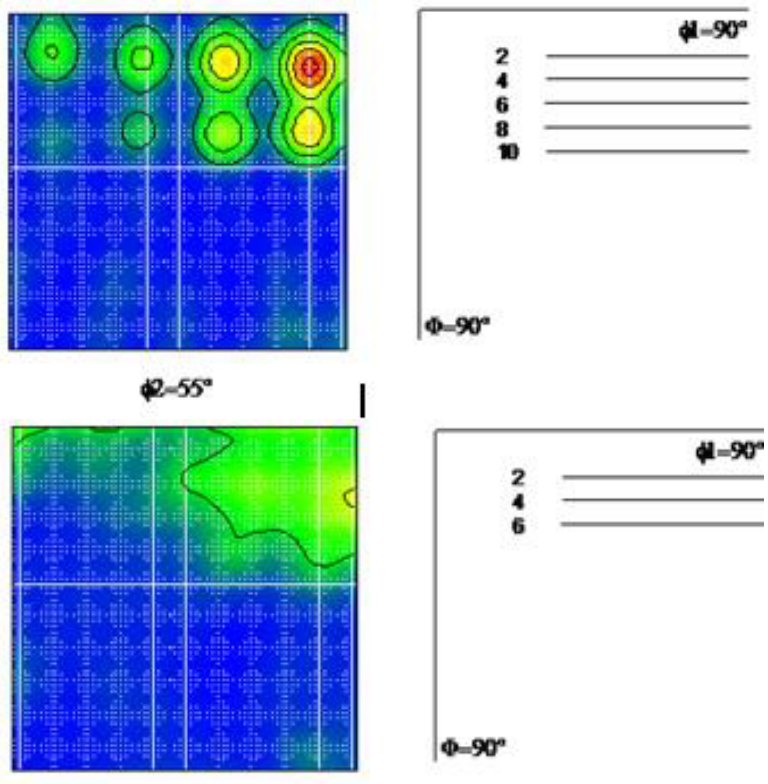

$2-55^{\circ}$

Figure 3. Contour lines of ODF cross section for fixed $h=0.5 \mu \mathrm{m}$, (a) for $\omega_{0}=20^{\circ}$ and (b) for $\omega_{0}=10^{\circ}$

Relying on the contour lines of ODF cross section (Fig. 3) we receive the sufficient maximums growing (almost twice) for $\omega_{0}=20^{\circ}$ versus ones for $\omega_{0}=5^{\circ}$ and $10^{\circ}$. Angles $\phi 1, \Phi, \phi 2$ (Fig. 3) correspond to Euler angles $\varphi, \theta, \psi$ respectively. Besides unsuitable meaning of $\omega_{0}$ may changed ODF character. This observation was also found out during the processing of model experiment results [5].

\section{Summary}

The comparison of PF and ODF calculation results for model and real EBSD experiment found out strong similarity and gave us the ability to use our model for study of choosing measurement parameters. For the single-phase strong textured specimen with small amount of boundaries the threshold disorientation angle should not be more than $10^{\circ}$ and the step size equal to half an average grain size is suitable. The step size being less than a quarter of average grain size can result in unjustifiably high time of measuring. Moreover, step size should not less a quarter of average grain size as it can result in unjustifiably high time of measuring in view of inessential result improvement.In general researchers need to carry out the corresponding modeling for each specimen with new characteristics and further model experiment with parameters varying to choose appropriate ones.In the future the improvement of experiment and specimen model as well as investigation of wider class of materials using the model developed are planned.

\section{References}

1. A. J. Schwartz, M. Kumar, B. L. Adams, and D. P. Field, Electron Backscatter Diffraction in Materials Science, 2nd. ed. Springer, New York, (2009).

2. E. Guilmeau, C. Henrist, T. S. Suzuki, Y. Sakka, D. Chateigner, D. Grossin, and B. Ouladiaf, ICOTOM 14, 179, (2005).

3. S. Piazolo, V. G. Sursaeva, and D. J. Prior, ICOTOM 14, 213 (2005).

4. M. V. Sypchenko, T. I. Savyolova, Cristallogr. Rep. 55 (4), 546 (2010).

5. A.O. Antonova, T. I. Savyolova, Comput. Math. Math. Phys. 55 (2), 317 (2015).

6. A.O. Antonova, T. I. Savyolova, Cristallogr. Rep. 61 (3), 1 (2016).

7. T. I. Savyolova, T. M. Ivanova, and M. V. Sypchenko, Methods for Solving Ill-Posed Problems in Texture Analysis and Their Applications, Mosk. Inzh._Fiz. Inst., Moscow, (2012) [in Russian].

8. N. Bozzolo, F. Gerspach, G. Sawina, and F. Wagner, J. Microscopy. 227, 275 (2007).

9. V.A. Gribkov, F.I. Grigoriev, B.A. Kalin, V.L. Yakushin, Advanced Radiation-Beam Technologies of Materials Treatment, Krugly God, Moscow, (2001). [in Russian]. 